American Journal of Environmental Sciences 3 (3): 175-182, 2007

ISSN 1553-345X

(C) 2007 Science Publications

\title{
Open ended-coaxial Cable Measurements of Saturated Sandy Soils
}

\author{
${ }^{1}$ Alberto Godio \\ ${ }^{1}$ DITAG - Politecnico di Torino, Corso Duca degli Abruzzi 24 - 10129 Torino, Italy
}

\begin{abstract}
The application of electromagnetic methods in the radar and micro-wave frequency band allows the fluid content of soils to be estimated; the heuristic or physically-based models permit to relate the observed electrical permittivity of the soils with the porosity and fluid saturation. The role of the effect of the free water and bound water in the overall electromagnetic behaviour of soils is discussed. The measurements of the complex electrical permittivity of samples with different fluid content were carried out using an open ended coaxial cable in the frequency range between 0.2 and 6 GHz. The data processing permitted to estimate the water content with good accuracy; the slight overestimate of the values with respect to the standard laboratory measures of the fluid content is due to the propagation of the experimental uncertainties and to the effect of the bound water.
\end{abstract}

Keywords: soil water content, electromagnetic, hydrocarbon contaminants

\section{INTRODUCTION}

The investigation of the electromagnetic properties of porous soils at low frequency ${ }^{[1,2]}$ and in the radio and microwave frequency range is increasing in the environmental sciences; the interest is justified by the TDR (Time Domain Reflectometry) and georadar (GPR) capability to estimate the water content and the possibility of a fast mapping on wide areas of the water content in the upper layer of the subsoil. Moreover the application of cross-hole radar in the soil sciences requires robust relationships between the observed parameters (electrical permittivity) and the porosity and water content ${ }^{[3,4]}$. The laboratory measurements of the dielectric permittivity of rocks and soils at different fluid saturation are widely diffused ${ }^{[5,6,7]}$. The application of TDR to estimate the hydrocarbon pollution is another challenge that should be analysed in $\operatorname{details}^{[8]}$.

In such a context a short discussion of the most diffused models to relate the electromagnetic parameters of soils with the fluid content is presented; the discussion considers the effect of the free water and bound water on the reliability of the predictive models of the electromagnetic behaviour of porous rocks. The bound water acts as a medium that reduce the overall polarizability of the molecules of the water; this decreases the values of the electrical permittivity of the water content. The effect is relevant for soils with high content of particles characterised by high specific surface where the bound water content is significant with respect to the total volume of interstitial water.

The paper deals with the laboratory measurements to the estimate the electrical permittivity of samples of sandy soils in dry condition and saturated with water and diesel oil; the measurements were carried out using a dielectric probe kit connected with a network analyser that permitted the spectrum of real and imaginary part of the permittivity to be recorded in the frequency range usually adopted in TDR and GPR applications ${ }^{[9]}$.

\section{MATERIALS AND METHODS}

Theoretical background: The electrical conductivity at the radar and microwave frequency is characterised by in-phase $\left(\sigma^{\prime}\right)$ and out of phase $\left(\sigma^{\prime \prime}\right)$ component according to the general form:

\section{$\sigma=\sigma^{\prime}-i \sigma^{\prime \prime} \quad$ [Siemens/meter]}

The permittivity indicates the polarizability of a material: as the frequency increases the polarisation response may lag behind the varying field; therefore the general form of permittivity involves an in-phase $\left(\varepsilon^{\prime}\right)$ and out of phase ( $\varepsilon$ ") component:

$$
\varepsilon=\varepsilon^{\prime}-i \varepsilon^{\prime \prime} \quad[\mathrm{Farad} / \mathrm{meter}]
$$

The parameters $\alpha$ and $\beta$ that are the attenuation and propagation constant:

$$
\begin{aligned}
& \alpha=\omega \cdot\left\{\left(\frac{\mu \varepsilon}{2}\right)\left[\left(1+\left(\frac{\sigma}{\omega \varepsilon}\right)^{2}\right)^{0.5}-1\right]\right\}^{0.5}(\mathrm{~Np} / \mathrm{m}) \\
& \beta=\omega \cdot\left\{\left(\frac{\mu \varepsilon}{2}\right)\left[\left(1+\left(\frac{\sigma}{\omega \varepsilon}\right)^{2}\right)^{0.5}+1\right]\right\}^{0.5}(\mathrm{rad} / \mathrm{m})
\end{aligned}
$$

The frequency dependence of $\alpha$ and $\beta$ is related to the frequency $(\omega)$ dependence of the constitutive parameters of the permittivity and conductivity; instead the magnetic permeability $\mu$ is equal to the permeability of free space for most non-magnetic rocks ${ }^{[10]}$.

The attenuation is related to a quality factor $(\mathrm{Q})$; this parameter indicates the energy stored to the energy

Corresponding Author: Alberto Godio - DITAG - Politecnico di Torino, Corso Duca degli Abruzzi 24 - 10129 Torino, Italy 
dissipated in a propagating wave; it can be determined from the equation:

$$
Q(\omega)=\frac{\omega}{2 c \alpha(\omega)}
$$

where $\mathrm{c}$ is the phase velocity. In radio wave propagation the $\mathrm{Q}$ parameter is closely related to the loss tangent:

$Q \approx 1 / \tan \delta$

Laboratory measurements show that dielectric permittivity is a general frequency dependent complex quantity for most earth materials. Attenuation measurements pointed out that frequency effect is usually a (quasi) linear function of frequency. The theoretical models, such as the Debye and the ColeCole model ${ }^{[11]}$ describe the frequency-dependence behaviour of the electrical permittivity of earth materials. The dielectric constants, $\varepsilon$ ' and $\varepsilon$ " depends on both frequency and temperature, the first of which is expressed explicitly in the Debye equations whilst temperature is introduced indirectly though other variables.

The electromagnetic behavior of porous soils is usually approximated considering the material as a mixture of solid matrix (the skeleton of the soil), water and air. In such a way some models permit to compute the electromagnetic constitutive parameters of the mixture from the properties and the volume fraction of each material.

The properties of the solid matrix are related to the primary rock; most rocks are characterised by real part of the electrical permittivity (relative) in the range between 4 and 9, while the imaginary part is often negligible. The electrical permittivity of water depends on the temperature and salinity; at standard condition the relative permittivity (real) is around 75-80.

The complexity of the physical and chemical reaction at the interface between water and solid skeleton have to be considered in the estimate of the electromagnetic behaviour of soils; the retention water (bound water) is strongly connected to the solid matrix and its polarizability is reduced in comparison with the polarizability of the free water. In the past, experiments were carried out to assess the effect of the bound water on the permittivity of the soil; Thorp ${ }^{[12]}$ considered two layers of bound water around the solid grain, the first with an average permittivity of 41 and the second one with a value of 66 . Sposito ${ }^{[13,14]}$ considered tree or four layers, as a function of mineralogical content of the soil, with an average value of 20. Dirksen and Dasberg $^{[15]}$ considered a value of 3.2 (as ice) for a mono-molecular layer of bound water around the grains. This value gradually increases with the distance from the surface of the grain. Wraith e Or ${ }^{[16]}$ described the effect of temperature due to the presence of bound water; they considered an average value of 12 .

Hasted and al. ${ }^{[1]}$ used two different models to demonstrate the effects of the electrical charge on the electrical permittivity of the water molecules; their concluded that three different layers can be considered with values of 18,72 and 78 . More recently, Dobson et al. ${ }^{[18]}$ and Friedman ${ }^{[19]}$ assumed a value of electrical permittivity of the bound water of 5.5.

A simple approximation of the values of permittivity with distance from the grain surface is:

$\varepsilon_{w}=\varepsilon_{f w} \cdot\left(1-e^{-x / \beta}\right)$

where $\varepsilon_{\mathrm{w}}$ is the permittivity of water as a function of the distance $\mathrm{x}, \varepsilon_{\mathrm{fw}}$ is the permittivity of free water (80) and $\beta$ is the thickness of the bound water layer (2.8 angstrom).

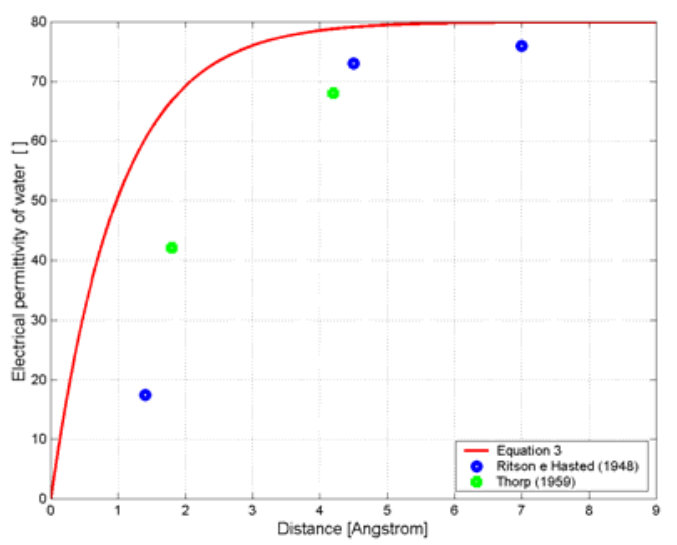

Fig.1: values of electrical permittivity of bound water according to formula 3

The average value of the bound water can be determined by the integration of the formula 3 :

$\varepsilon_{w}(A v)=\left[\left(x+\beta e^{-x / \beta}-\beta\right) \cdot \varepsilon_{f w}\right] / x$

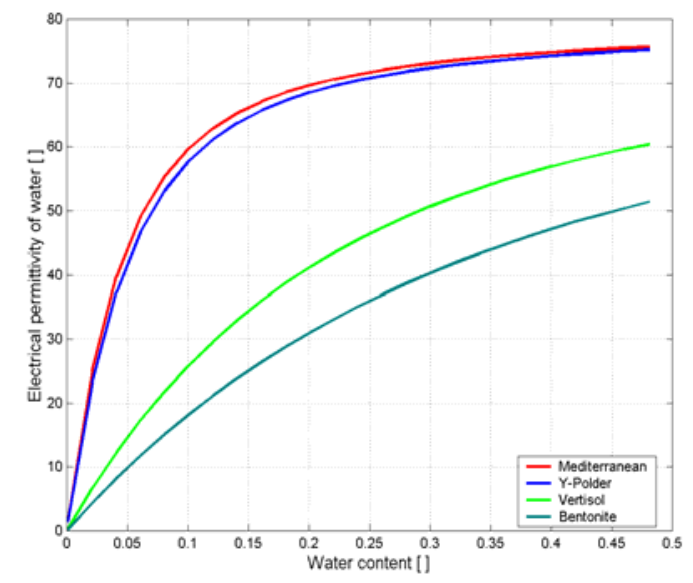

Fig.2: the permittivity of water as a function of the mineralogical content of soils according to the formula (5); soils with high clay contents (Vertisol and Bentonite) provides for the lowest values of water permittivity that doesn't reach the conventional values of the free water (80). 
The relationship 4 can be written according to the water content instead of the geometrical parameters $\mathrm{x}$ and $\beta$ :

$$
\varepsilon_{w}(A v)=\left\lfloor\left(\theta+\theta_{f w} \cdot e^{-x / \beta}-\theta_{b w}\right) \cdot \varepsilon_{f w}\right\rfloor / \theta
$$

where $\theta$ is the free water content, $\theta_{\mathrm{bw}}$ is the bound water content.

A similar approach to compute the effect of the bound water considers the thickness of the water shell related to the specific surface and bulk density of the soil:

$$
d w=\theta /\left(\rho_{b} \cdot S_{S A}\right)
$$

The permittivity of the water is the harmonic mean:

$$
d w=(1 / d w) \cdot \int(1 / \varepsilon(x)) \cdot d x
$$

and the water permittivity is:

$\varepsilon_{w}=\left(d w \varepsilon_{\max }\right) /\left\{d w+\ln \left[\left(\varepsilon_{\max }-\left(\varepsilon_{\max }-\varepsilon_{\min }\right) \cdot e^{-\lambda d w}\right) / \varepsilon_{\min }\right]\right\}$

where $\varepsilon_{\max }$ is the free water permittivity (80) and $\varepsilon_{\min }$ is the bound water permittivity (5.5) and $\lambda=1$ Angstrom $\left(10^{-10} \mathrm{~m}\right)$.

The dielectric response of wetted porous media under changing temperature may be useful in estimating the specific surface area $\left(\mathrm{m}^{2} \mathrm{~kg}^{-1}\right)$, because of the fundamental relationship between bound water and solid surface area ${ }^{[20]}$. Expressions have been derived for describing the combined bound-plus freewater dielectric constant based on the surface area and bulk density and including the temperature dependence ${ }^{[21]}$. Therefore the permittivity of water in the pore volume of the soils is strongly dependents to the mineralogical content (specific surface), the pore dimensions and the distance of the water molecules with respect to the grain surface.

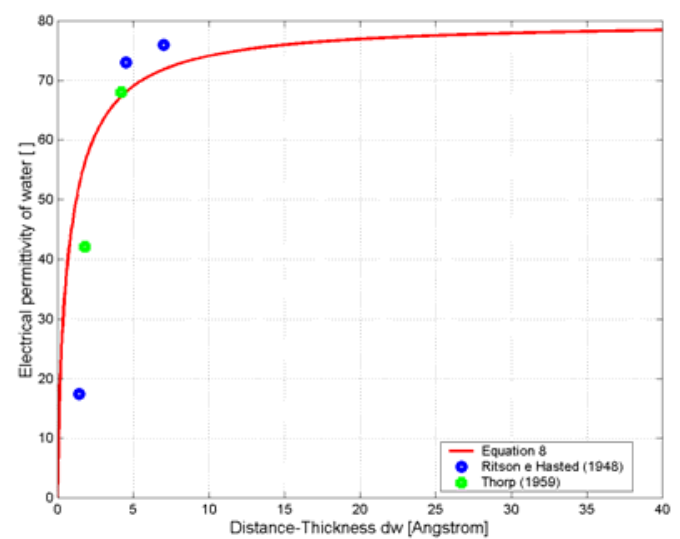

Fig.3: water permittivity versus the distance with the solid grain, according to the relationship (8)

The hydrocarbon contaminant in soils acts as a non-miscible fluid phase dispersed in the pore volume; a simplified approach considers the displacement of water by the hydrocarbon during the contamination or the displacement of the hydrocarbon phase by the water, for instance in the vadose zone due to the seasonal fluctuations of the water table or to the water infiltration from the surface.

The electromagnetic behaviour of hydrocarbon polluted soils can be assimilated to a mixture of different phases; in saturated conditions the solid grains are coated by the bound water and the hydrocarbon particles are coated by water with electrochemical interaction with the hydrocarbons; finally the free water characterised the other non miscible phase. The more complex models take into account the shape and distribution of the different non-miscible phases.

In most cases the hydrocarbon is dispersed in the pore volume as free phase hydrocarbon; a small fraction is dissolved in the free water or in the bound water. Due to the reduced volume of the dissolved hydrocarbon, the water permittivity is not modified; instead the permittivity of the mixture is modified according to the volume fraction and the electromagnetic properties of the hydrocarbon. The electromagnetic properties of hydrocarbons are related to the polar or non-polar behavior of the molecules; light contaminants such as diesel oil, gasoline etc. are usually non-polar materials; they are characterised by low real part of the electrical permittivity (in the range between 2 and 3). Polar hydrocarbon (such as PCE and TCE) are characterised by higher permittivity value, in the range between 1113. The imaginary part is usually very low and often can be neglected in the evaluation of the electromagnetic response of contaminated soils.

Empirical or theoretically based approaches are used to investigate the relationship between the fluid content and the electromagnetic response of soils. Most of these models are heuristic or semi-empirical or based on statistical evaluation (Topp model); other ones preserve the importance of the grain size and shape and take the textural effects of the soil in to account ${ }^{[22]}$.

The empirical approach, e.g. suggested by Topp ${ }^{[23]}$ can be used to calculate the volumetric water content $(\theta)$ in sandy soil from measurements of the dielectric constant of the soil $(\varepsilon)$ :

$\theta=-5,3 \times 10^{-2}+2,92 \times 10^{-2} \varepsilon-5,5 \times 10^{-4} \varepsilon^{2}+4,3 \times 10^{-6} \varepsilon^{3}$

On the other hand, a simple mixing model can be adopted for describing the behaviour of the electrical permittivity of a mixture of solid matrix, air and water ${ }^{[24]}$. The following relationship between dielectric constant of a two phase mixture (soil particles-water or soil particles-air) results for a low-dispersive medium with a low loss factor $(\tan \delta<<1)$ :

$\sqrt{\varepsilon}=\phi \sqrt{\varepsilon_{w}}+(1-\phi) \sqrt{\varepsilon_{m}}$

where $\varepsilon$ is the dielectric constant of the mixture, $\varepsilon_{\mathrm{w}}$ is the dielectric constant of the wetting phase and $\varepsilon_{\mathrm{m}}$ is the dielectric constant of the wetted phase, $\phi$ is the medium porosity. This is the well know CRIM model (Complex Refractive Index Method), which is useful when the fluid content in a porous medium must be 
estimated starting from the electromagnetic measurements at radar and microwave frequencies.

The mixing formulas are a first degree of approximation of the dielectric behaviour of the porous medium; accurate models introduce at least one further variable which characterise the shape and orientation of the particles of the mixture. For air-solid mixture or for water-solid mixture the following formula can be adopted:

$\left(\frac{\varepsilon_{m}-1}{\varepsilon_{m}+u}\right)=p \cdot\left(\frac{\varepsilon_{1}-1}{\varepsilon_{1}+u}\right)+(1-p) \cdot\left(\frac{\varepsilon_{2}-1}{\varepsilon_{2}+u}\right)$

where $\varepsilon_{\mathrm{m}}$ is the complex permittivity of the mixture and $\varepsilon_{1}$ and $\varepsilon_{2}$ are the permittivity of two separate media (airice), $p$ is the fraction of the total volume occupied by medium 1; $\mathrm{u}$ is the Formzahl coefficient that depends on the structure of the material. The physical meanings of the coefficient was discussed by Evans ${ }^{[25]}$. Numerical values of the Formzahl in practice can range from 2 (for an aggregate of spherical particles) to infinity (for highly elongated particles oriented essentially parallel).

Apparatus: An open-ended coaxial line was used to measure the permittivity of lossy and low-lossy dielectric at radio and microwave frequency. The device if adequately optimised and used with accurate device for measuring the reflection coefficient, the sensor is capable of providing good accuracy in estimate effective permittivity of rocks.

The use of a dielectric probe kit and a network analyser permits a fast measurement of the complex permittivity parameters of rock samples in the wide frequency range (e.g. from $0.2 \mathrm{GHz}$ up to $20 \mathrm{GHz}$ ). Measurements of soils with different moisture contents are widely reported ${ }^{[26,27]}$; Peplincki ${ }^{[28]}$ used a dielectric probe device in the frequency range between 0.3 and $1.3 \mathrm{GHz}$. Wensink ${ }^{[29]}$ reported the results of the effective dielectric permittivity as a function of the frequency in the range $1-3000 \mathrm{MHz}$ with different water contents using a coaxial cable connected with a network analyser. The permittivity values were determined by measuring the reflection and transmission coefficients. Complex permittivity has been determined for soil mixed with organic compounds in the bandwidth $0.2-1.3 \mathrm{GHz}$ using a network analyser with a dielectric probe HP-85070 A by Santamarina and Fam $^{[30]}$.

An exhaustive analysis and comparison of methods to measure the dielectric permittivity of rocks is presented by Turner $^{[31]}$; they pointed out that the coaxial cell technique is one of the most accurate among different approaches, giving the most reliable results in terms of determination both the real and imaginary part of the complex permittivity. However the preparation of specimens to be tested is rather complicated and time consuming. On the other hand, the Open Ended Coaxial cable technique (OECC) gives less accurate results but the specimen preparation is very easy.

Measurements: The laboratory measurements of permittivity (real and imaginary part) in the frequency range of $200 \mathrm{MHz}$ to $6 \mathrm{GHz}$ have been performed on samples of sandy soils in water saturated, oil saturated and dry conditions. The equipment used was a HP 85070B dielectric probe kit connected to a HP network analyser, under control of a personal computer by a GPIB (IEEE488 standard). The measurements were carried out by the contact between the surface of the sample and the probe; for liquids (water and alcohols) measurements have been performed by immersing the probe into specimen. The dielectric parameters of the material (real and imaginary part) can be determined from the reflection coefficients at the probe-sample interface, according to the theoretical approach suggested by Stuchly et al. ${ }^{[32]}$.

The system was calibrated with open and short measurements on water or non polar liquids, such as alcohols. Fluids were tested by submerging the probe into the fluid keeping a minimum distance of about 2 $\mathrm{cm}$ from the container bottom. For rock specimens the accuracy of measure is related to the quality of the surface contact between the probe and material under testing. For mixture materials, the measurements were repeated both with the probe on the surface both with the probe immersed into the mixture: no remarkable differences were observed.
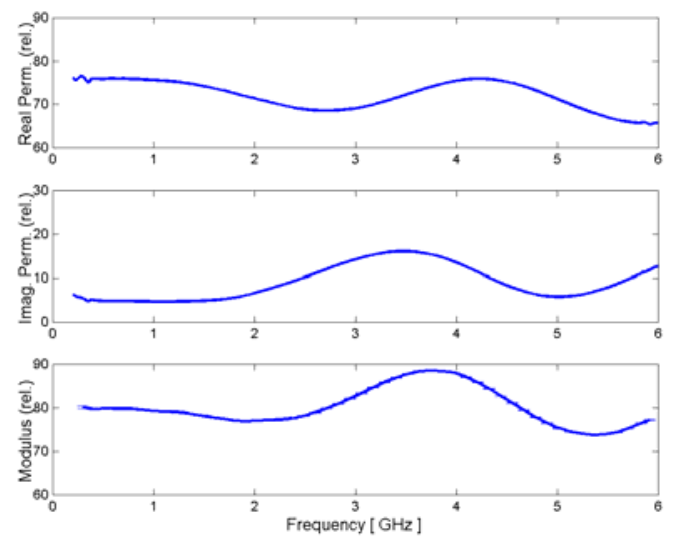

Fig.4: Sample water - top) real part of the dielectric permittivity; centre) imaginary part; bottom) modulus of the permittivity.

The accuracy in the estimate of the dielectric permittivity depends on the frequency of the external electromagnetic field; for samples with low dielectric constant (dry sand, oil and sand saturated with diesel oil) and at for frequency below $1 \mathrm{GHz}$, the typical accuracy is in the range of $5-20 \%$. The loss factor and the attenuation rate of the materials can be calculated from the real and imaginary part of the dielectric 
constant. Laboratory measurements on the porosity and size distribution of the medium were also carried out; the results between the electromagnetic measurements and standard measurements of porosity and fluid contents are compared.

\section{RESULTS AND DISCUSSION}

The laboratory measurements of the electromagnetic parameters have been useful to estimate the reliability of the predicting models in evaluating the electromagnetic behaviour of the sandy material in dry and saturated conditions.

It can be useful to analyse the experimental inaccuracy propagation of the results in the estimate of the fluid content of the samples; the overall inaccuracies are due to:

1. the network analyser error sources and the dielectric error source usually less than 3-4\%;

2. the typical probe accuracy, which depends on the frequency and electromagnetic property of the sample; the accuracy is usually low at low frequency and for low dielectric values of the material under test, the accuracy increase at high frequency $(>2 \mathrm{GHz})$ and for dielectric values higher than 30 .

The spectrum for different water-saturated samples is reported in figures 5-8; the plots depict the trend of the real, imaginary part and the modulus of the electrical permittivity of the water sand mixtures. The trend of the imaginary part is affected by a deviceresonant effect, that is visible in correspondence of the peak of the spectrum, at approximately the frequency of 3-3.5 GHz.

The effect of ionic conductivity is also present in the data; this is associated to the electromagnetic loss of the material due to surface-conductivity effect. It can be expressed as a function of the dielectric loss at very high frequency $\left(\varepsilon^{\prime \prime}{ }_{\text {inf }}\right)$ and the conductivity $(\sigma)$ according to the following relationship proposed by Nettelblady and Niklasson ${ }^{[33]}$ :

$\varepsilon^{\prime \prime}=\varepsilon_{\text {inf }}^{\prime \prime}+\frac{\sigma}{\omega \cdot \varepsilon_{0}}$

At low frequencies, the overall conductivity is controlled by different conduction mechanisms, where ionic conductivity is the most important in mixture materials. The imaginary part of the electrical permittivity is governed by the influence of the electrolytic conduction caused by free ions which exist in the presence of a solvent (water). At low frequency the effect of ionic conductivity is inversely proportional to frequency; the curve of imaginary versus frequency exhibits a slope of $1 / \mathrm{f}$. For sample in dry condition, a good fitting between the experimental curve of the imaginary part and the equation (12) is given for the conductivity values in the range between 0.01 and 0.02 $\mathrm{S} / \mathrm{m}$..
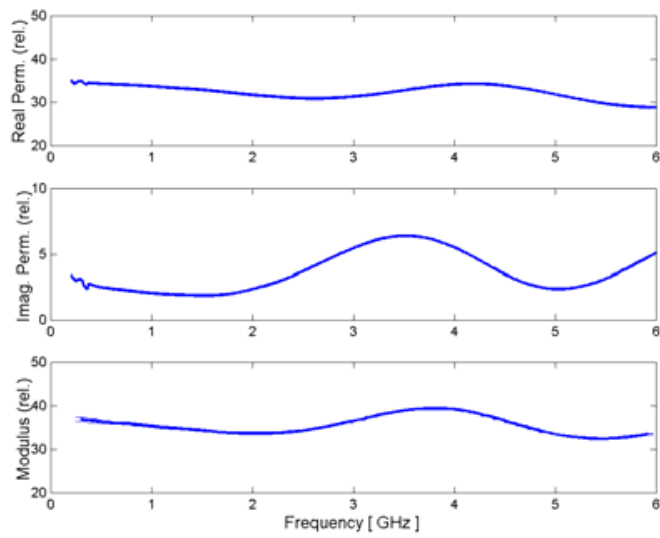

Fig.5: Sample Sand \# 1 (water saturated) - top) real part of the dielectric permittivity; centre) imaginary part; bottom) modulus of the permittivity.
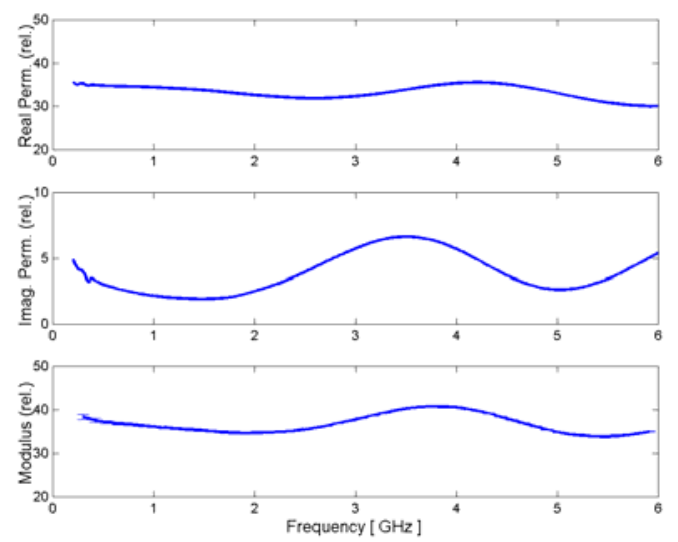

Fig.6: Sample Sand \# 3 (water saturated) - top) real part of the dielectric permittivity; centre) imaginary part; bottom) modulus of the permittivity.

For samples saturated with diesel oil the effect of the ionic conductivity is less pronounced; at low frequency the response can be affected by other mechanisms such as the interfacial effects or the space charge polarization. The conditions for the existence of a dielectric relaxation due to Maxwell-Wagner effect in heterogeneous binary mixtures are derived from the general laws of electromagnetism by Clausse ${ }^{[34]}$. A dielectric relaxation can occur only if the timeconstants of the components of the system are different from each other and different from zero as well, whichever be the geometry such as water-in-oil, oil-inwater. Mixtures of materials with electrically conducting regions (e.g. the bound water) that are not in contact with each other separated by non conducting region exhibit the Maxwell-Wagner effect at low frequencies; it means that the diesel oil partially displaces the free water of the sandy soil, coated the grain materials and acts as insulator between the water thin layers. However in the present paper we consider 
that the role of the interfacial polarisation (Maxwell Wagner) effect, which results from interfacial phenomena in inhomogeneous materials, is negligible at the microwave frequencies; theoretical and experimental studies have showed that the M-W effect can be observed in soil at frequency lower than 100 $\mathrm{MHz}^{[35]}$, out of the frequency range of the measures under discussion.
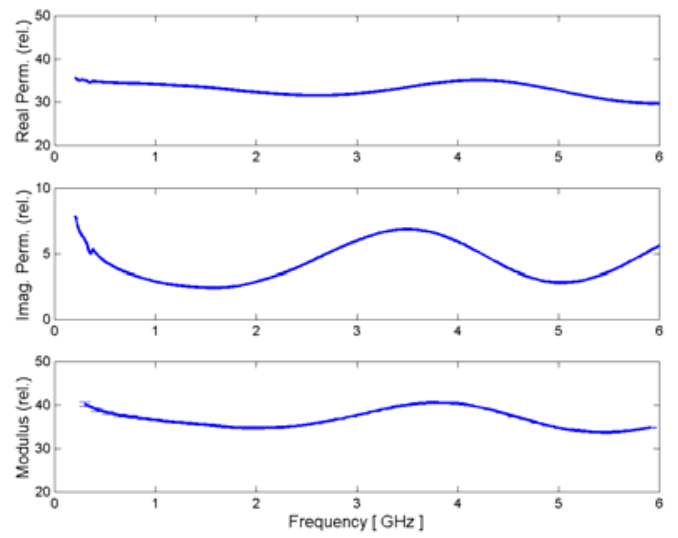

Fig.7: Sample Sand \# 4 (water saturated) - top) real part of the dielectric permittivity; centre) imaginary part; bottom) modulus of the permittivity.
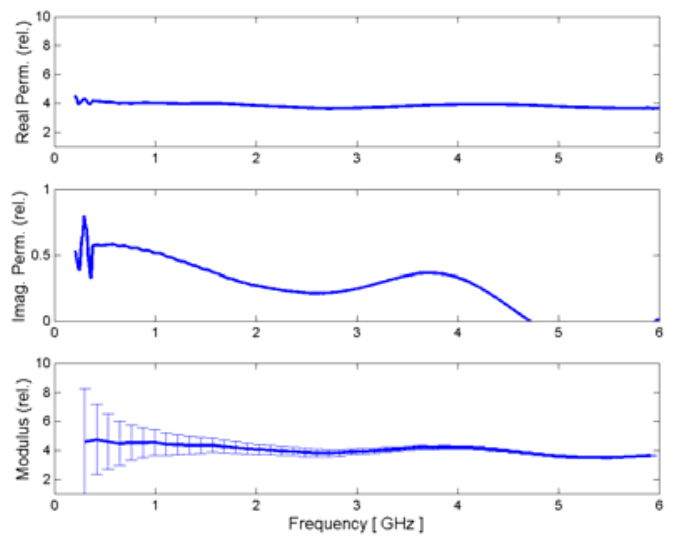

Fig.8: Sample Sand \# 3 (diesel oil saturated) - top) real part of the dielectric permittivity; centre) imaginary part; bottom) modulus of the permittivity.

The general method of getting formulas for propagating errors involves the total differential of the function. We treat the $\mathrm{d} \phi=\Delta \phi$ as the error or standard deviation in the porosity evaluation, and likewise for the other differentials.

The formula (10) can be modified for the 4-phases systems (solid matrix-free water-bound water-air); the model considers the time of propagation of the electromagnetic wave in a multiphase porous medium starting form the time average equation, that converted in electrical permittivity, yields: $\varepsilon_{m}=\left[(1-\phi) \cdot \varepsilon_{s}^{0.5}+(\phi-\vartheta) \cdot \varepsilon_{a}^{0.5}+\left(\theta-\theta_{b w}\right) \cdot \varepsilon_{w}^{0.5}+\theta_{b w} \cdot \varepsilon_{b w}^{0.5}\right]^{2}$

where $\varepsilon_{\mathrm{m}}$ is the permittivity of the solid-air-waterbound water mixture; $\phi$ is the porosity, $\theta$ is the water content; $\theta_{\mathrm{bw}}$ is the bound water content; $\varepsilon_{\mathrm{s}}$ is the solidgrain permittivity, $\varepsilon_{\mathrm{a}}$ is the air permittivity; $\varepsilon_{\mathrm{w}}$ the water permittivity and $\varepsilon_{\mathrm{bw}}$ is the bound water permittivity.

A simple case yields when the measures at different fluid content performed on the same porous medium are available; for instance if $\varepsilon_{\text {dry }}$ and $\varepsilon_{\text {sat }}$ are the permittivity of the sandy samples in dry and saturated condition, the following mixing formula can be written (assuming that $\theta_{\mathrm{bw}}$ can be neglected):

$\varepsilon_{d r y}^{0.5}=(1-\phi) \cdot \varepsilon_{s}^{0.5}+(\phi) \cdot \varepsilon_{a i r}^{0.5}$

and in saturated condition

$\varepsilon_{d r y}^{0.5}=(1-\phi) \cdot \varepsilon_{s}^{0.5}+(\phi) \cdot \varepsilon_{w}^{0.5}$

For a fully saturated medium the porosity values is equal to the values of the fluid content:

$\phi=\frac{\varepsilon_{s a t}^{0.5}-\varepsilon_{d r y}^{0.5}}{\varepsilon_{w}^{0.5}-\varepsilon_{a}^{0.5}}=\frac{\varepsilon_{s a t}^{0.5}-\varepsilon_{d r y}^{0.5}}{\varepsilon_{w}^{0.5}-1}$

The propagation error on the estimate of the porosity value is:

$\Delta \phi=\left|\frac{\partial \phi}{\partial \varepsilon_{s a t}}\right| \cdot \Delta \varepsilon_{s a t}+\left|\frac{\partial \phi}{\partial \varepsilon_{d r y}}\right| \cdot \Delta \varepsilon_{d r y}+\left|\frac{\partial \phi}{\partial \varepsilon_{w}}\right| \cdot \Delta \varepsilon_{b w}$

To minimize the effect of the surface conductivity and the error of the network that are usually most relevant at the low frequency, the porosity and fluid content values have been determined at the reference frequency of $2 \mathrm{GHz}$ and listed in table 1 .

The sensitivity determines the importance of each experimental parameters in the estimate of the fluid content; at the reference values reported in table 1 , the value of the dielectric permittivity in dry condition contributes for more than $70 \%$ to the overall sensitivity; the water permittivity and the permittivity of the sample in saturated condition affect the sensitivity for the $10 \%$ and $20 \%$ respectively. Unfortunately the permittivity values of soils in dry condition are very sensitive to the experimental inaccuracies both according to the laboratory measurements here discussed using the dielectric probe system, and adopting the in situ system such as the TDR devices. This aspect strongly affects the accuracy in the estimate of values of fluid content in the selected samples.

For samples saturated with diesel oil, the sensitivity is affected for the $40-45 \%$ from the term in saturated condition; the term in dry condition contribute for the $45 \%$ and the $10-15 \%$ is due to the estimate of the diesel oil electromagnetic behavior. This means that the model is not sensitive to the electromagnetic properties of the hydrocarbon itself.

The overestimate of the values of water content derived by the open ended coaxial measurements with respect to the values observed by the standard 
gravimetric sampling is partially justified by the effect of bound water on the permittivity model.

The open-ended coaxial line has some disadvantages in practical use on porous materials: the sensitive area is relatively small, and the sensor is soiled during each measurement. The dielectric data are only an intermediate result for the determination of the composition values. They are calculated from the measured reflection factors at the aperture of the openended coaxial cable.
Accurate calibration and differential measurement between material under test and the response of a known substance can mitigate the effect of propagation of the experimental inaccuracies on the model response. For samples of soils which can be considered as mixture porous media, the fluid content can be estimated by the difference between the measures in dry and in saturated condition. This leads to an inaccuracy of $10-15 \%$ in the estimate of water content. Moreover, the study shows that the approach has a low accuracy in the estimate of the hydrocarbon fluid content.

\begin{tabular}{|c|c|c|c|c|c|c|}
\hline Medium & Samples & $\begin{array}{l}\text { Dielectric. } \\
\text { Real Part }\end{array}$ & $\begin{array}{l}\text { Dielectric } \\
\text { Imag. Part }\end{array}$ & $\begin{array}{c}\text { Abs. Dielectric } \\
\text { Permittivity } \\
\operatorname{Abs}\left(\varepsilon^{\prime}+\mathrm{j} \varepsilon^{\prime \prime}\right)\end{array}$ & $\begin{array}{c}\text { Measured } \\
\text { Volumetric fluid } \\
\text { content } \\
{\left[\mathrm{m}^{3} / \mathrm{m}^{3}\right]}\end{array}$ & $\begin{array}{c}\text { Estimated Vol. } \\
\text { fluid content } \\
{\left[\mathrm{m}^{3} / \mathrm{m}^{3}\right]}\end{array}$ \\
\hline Water - Sample 1 & A1b & 71.3 & 6.0 & $77.0 \pm 1$ & & \\
\hline Water - Sample 2 & $\mathrm{~A} 2 \mathrm{~b}$ & 70.1 & 6.5 & $75.3 \pm 1$ & & \\
\hline Diesel Oil & G1b & 2.2 & 0.04 & $2.2 \pm 1$ & & \\
\hline Dry Sand \#1 - Siro & $\mathrm{S} 1 \mathrm{~b}$ & 3.0 & 0.10 & $3.0 \pm 1$ & & \\
\hline Dry Sand \#2 - France & $\mathrm{S} 3 \mathrm{~b}$ & 2.6 & 0.12 & $2.6 \pm 1$ & & \\
\hline Dry Sand \#3 - Ticino & $\mathrm{S} 2 \mathrm{~b}$ & 2.7 & 0.11 & $2.7 \pm 1$ & & \\
\hline Dry Sand \#4 - fine & $\mathrm{S} 4 \mathrm{~b}$ & 2.6 & 0.20 & $2.8 \pm 1$ & & \\
\hline Dry Sand \#5 - coarse & $\mathrm{S} 5 \mathrm{~b}$ & 2.6 & 0.36 & $2.9 \pm 1$ & & \\
\hline Water Saturated Sand \#1 & S6a & 31.7 & 2.30 & $33.7 \pm 0.2$ & $0.50 \pm 0.02$ & $0.52 \pm 0.05$ \\
\hline Water Saturated Sand \#2 & $\mathrm{S} 7 \mathrm{a}$ & 29.3 & 2.05 & $30.9 \pm 0.2$ & $0.40 \pm 0.02$ & $0.51 \pm 0.05$ \\
\hline Water Saturated Sand \#3 & $\mathrm{S} 8 \mathrm{~b}$ & 34.3 & 2.57 & $36.4 \pm 0.2$ & $0.40 \pm 0.02$ & $0.56 \pm 0.05$ \\
\hline Water Saturated Sand \#4 & S9a & 32.3 & 2.84 & $34.7 \pm 0.2$ & $0.47 \pm 0.02$ & $0.54 \pm 0.05$ \\
\hline Oil Saturated Sand \#1 & $\mathrm{S} 12 \mathrm{~b}$ & 3.3 & 0.03 & $3.3 \pm 1.0$ & & - \\
\hline Oil Saturated Sand \#2 & $\mathrm{S} 10 \mathrm{a}$ & 3.5 & 0.19 & $3.7 \pm 1.0$ & $0.40 \pm 0.02$ & $0.64 \pm 1.6$ \\
\hline Oil Saturated Sand \#3 & $\mathrm{S} 11 \mathrm{~b}$ & 3.8 & 0.27 & $4.0 \pm 1.0$ & $0.40 \pm 0.02$ & $0.75 \pm 1.6$ \\
\hline
\end{tabular}

\section{REFERENCES}

1. Slater, L. D. and D. Lesmes, 2002. IP interpretation in environmental investigations. Geophysics, 67: 77-88.

2. Godio, A. and M. Naldi, 2003. Two-dimensional electrical imaging for detection of hydrocarbon contaminants. Near Surf. Geophys., 1: 131-137.

3. Boniger, U, J. Tronicke, K. Holliger and A. Becht., 2006. Multi-offset vertical radar profiling for subsurface reflection imaging. Journal of Environmental and Engineering Geophysics, 11: 289-298.

4. Cassiani, G., C. Strobbia and L. Gallotti, 2004. Vertical radar profiles for the characterization of deep vadose zones. Vadose Zone Journ., 3: 10931105.

5. Wensink, W. A., 1993. Dielectric properties of wet soils in the frequency range 1-3000 $\mathrm{MHz}$. Geophys. Prosp., Eur. Assn. Geosci. Eng., 41:671696.
6. Hoekstra, P. and A. Delaney, 1979. Dielectric properties of soil at UHF and microwave frequencies, J. Geoph. Res., 72: 5101-5115.

7. Peplincki, N.R., F.T. Ulaby and M.C. Dobson, 1995. Dielectric properties of soils in the 0.3-1.3 $\mathrm{GHz}$ range, IEEE Trans. On Geoscience and Remote Sensing, 33: 803-807.

8. Persson, M. and R. Berndtsson, 2002. Measuring non-aqueous phase liquid saturation in soil using time domain reflectometry, Water Resources Research, 38: 1064-1072.

9. Friel, R. and D.Or, 1999. Frequency analysis of time-domain reflectometry (TDR) with application to dielectric spectroscopy of soil constituents, Geophysics, 64: 1-12.

10. Turner, G., A. F. Siggins, and L. D. Hunt, 1993. Ground-penetrating radar - Will it clear the haze at your site?, 10th Geophysical Conference, Austr. Soc. Expl. Geophys., 24: 819-832.

11. Cole, K. S. and Cole R. H., 1941. Dispersion and Absorption in Dielectrics I. Alternating Current Characteristics, J. Chem. Phys., 9: 341. 
12. Thorp, J.M.,1959. The dielectric behaviour of vapours adsorbed on porous solids, Transaction of the Faraday Society, 55: 442-454.

13. Sposito, G., 1984. The Surface Chemistry of Soils. (Oxford University Press: New York.).

14. Sposito, G., 1989. The Chemistry of Soils. (Oxford University Press: New York.).

15. Dirksen, C. and S. Dasberg, 1993. Improved calibration of time domain reflectometry for soil water content measurements, Soil Sci. Soc. Am. J., 57: 660-667.

16. Wraith, J.M. and D. Or, 1999. Temperature effects on time domain reflectometry measurement of soil bulk dielectric constant: experimental evidence and hypothesis development, Water Resources Research, 35: 361-369.

17. Hasted, J. B., D. M. Ritson and C. H. Collie, 1948. Dielectric properties of aqueous ionic solutions, J. Chem. Phys., 16: 1.

18. Dobson, M.C., F.T. Ulaby, M.T. Hallikainen and M.A. El-Rayes, 1985. Microwave dielectric behavior of wet soil-part II: dielectric mixing models; IEEE Transaction on Geoscience and Remote Sensing GE-23(1): 35-46.

19. Friedman, S.P., 1998. A saturation degreedependent composite spheres model for describing the effective dielectric constant of unsaturated porous media, Water Resources Research, $11,: 2949-2961$.

20. Or, D. and J.M Wraith. 1999. Temperature effects on soil bulk dielectric permittivity measured by time domain reflectometry: a physical model, Water Resources Research, 35: 371-383.

21. Jones, S.B. and D. Or, 2001. Surface area, geometrical and configurational effects on permittivity of porous media, Journal of NonCrystalline Solids, 305: 247-254

22. Hanai, T., 1969. Electrical properties of emulsion, in Sherman, P. Edition: Emulsion Science, Academic Press.
23. Topp, G.C., J. L.Davis and A. P. Annan, 1980. Electromagnetic determination of soil water content: measurements in coaxial transmission lines, Water Resources Research, 16: 574-582.

24. Birchak, J.R., C.G. Gardner, J.E. Hipp and J.M. Victor, 1974. High dielectric constant microwave probes for sensing soil moisture, Proc. IEEE, 62: 93-98.

25. Evans, S., 1965. Dielectric properties of ice and snow. J. Glaciology, 5: 773-792.

26. Hipp, J.E., 1974. Soil electromagnetic parameters as functions of frequency, soil density and soil moisture, Proc. IEEE, 62: 98-103.

27. Taherian, M. R., W. E. Kenyon, and K. A. Safinya, 1990. Measurement of dielectric response of watersaturated rocks, Geophysics, 55: 1530-1541.

28. see $[7]$.

29. see [ 5 ].

30. Santamarina, J.C. and M. Fam, 1997. Dielectric Permittivity of Soils Mixed with Organic and Inorganic Fluids $(0.02 \mathrm{GHz}$ to $1.30 \mathrm{GHz})$, J. Env. \& Engineering Geophysics, 2: 37-52.

31. see [ 10$]$

32. Stuchly, M., M. Brady, S. Stuchly and G. Gajda, 1982. Equivalent Circuit of an Open-Ended Coaxial Line in a Lossy Dielectric, IEEE Transactions on Instrumentation and Measurement, IM-31: 116-119.

33. Nettelblady B and G.A. Niklasson, 1996. The dielectric dispersion of liquid-filled porous sintered materials. J. Phys. Condens. Matter, 8: 2781-2790.

34. Clausse, M., 1975. Theoretical conditions for the existence of a dielectric relaxation due to MaxwellWagner effect in heterogeneous binary systems and especially in emulsions, Colloid \& Polymer Science, 253m: 1020-1024.

35. Hilhorst, M.,A., 1998. Dielectric characterisation of soil. WAU dissertation no. 2386. 